TRANSACTIONS OF THE

AMERICAN MATHEMATICAL SOCIETY

Volume 355, Number 12, Pages 4755-4765

S 0002-9947(03)03101-5

Article electronically published on July 24, 2003

\title{
A BAIRE'S CATEGORY METHOD FOR THE DIRICHLET PROBLEM OF QUASIREGULAR MAPPINGS
}

\author{
BAISHENG YAN
}

\begin{abstract}
We adopt the idea of Baire's category method as presented in a series of papers by Dacorogna and Marcellini to study the boundary value problem for quasiregular mappings in space. Our main result is to prove that for any $\epsilon>0$ and any piece-wise affine map $\varphi \in W^{1, n}\left(\Omega ; \mathbf{R}^{n}\right)$ with $|D \varphi(x)|^{n} \leq$ $L \operatorname{det} D \varphi(x)$ for almost every $x \in \Omega$ there exists a map $u \in W^{1, n}\left(\Omega ; \mathbf{R}^{n}\right)$ such that

$$
\left\{\begin{array}{l}
|D u(x)|^{n}=L \operatorname{det} D u(x) \quad \text { a.e. } x \in \Omega, \\
\left.u\right|_{\partial \Omega}=\varphi, \quad\|u-\varphi\|_{L^{n}(\Omega)}<\epsilon .
\end{array}\right.
$$

The theorems of Dacorogna and Marcellini do not directly apply to our result since the involved sets are unbounded. Our proof is elementary and does not require any notion of polyconvexity, quasiconvexity or rank-one convexity in the vectorial calculus of variations, as required in the papers by the quoted authors.
\end{abstract}

\section{INTRODUCTION}

Given $L \geq 1$, we consider the following Dirichlet problem of first-order partial differential equations:

$$
\begin{cases}|D u(x)|^{n}=L \operatorname{det} D u(x), & x \in \Omega, \\ u(x)=\varphi(x), & x \in \partial \Omega,\end{cases}
$$

where $\Omega$ is a bounded open set in $\mathbf{R}^{n}$ with boundary $\partial \Omega, u: \Omega \rightarrow \mathbf{R}^{n}, D u(x)$ is the Jacobi matrix of $u$ and $|D u(x)|$ uses the matrix operator norm (see (1.6) below). Here $\varphi$ is a given map.

In this paper, we study solutions of (1.1) that only have certain weak derivatives. Throughout the paper, we assume solutions $u$ to be in some Sobolev space, that is, $u \in W^{1, p}\left(\Omega ; \mathbf{R}^{n}\right)$, and thus the equation in (1.1) is required to be satisfied only for almost every $x \in \Omega$ and the boundary condition is understood in the sense of Sobolev functions.

Following Iwaniec [6], the weakly L-quasiregular mappings are defined to be mappings $u \in W_{\text {loc }}^{1, p}\left(\Omega ; \mathbf{R}^{n}\right)$, with some $p \geq 1$, that satisfy

$$
|D u(x)|^{n} \leq L \operatorname{det} D u(x), \quad \text { a.e. } x \in \Omega .
$$

Therefore (1.1) can be considered as a Dirichlet problem for a special class of weakly quasiregular mappings. If $p \geq n$, the weakly quasiregular mappings in $W^{1, p}\left(\Omega ; \mathbf{R}^{n}\right)$ are the usual quasiregular mappings, which are also called mappings of bounded

Received by the editors January 25, 2001.

2000 Mathematics Subject Classification. Primary 30C65, 35F30, 49J30.

Key words and phrases. Baire's category method, Dirichlet problem, quasiregular mappings. 
distortion [9. A classical Liouville's theorem asserts that any usual 1-quasiregular mapping is a restriction of Möbius transformation on $\Omega$; thus there are not too many of them. So, in this paper we restrict ourselves to the $L$-quasiregular mappings with $L>1$.

In Yan 10, 11, 12, we studied the boundary value problem for weakly $L$ quasiregular mappings in $W^{1, p}\left(\Omega ; \mathbf{R}^{n}\right)$ with $1 \leq p<\frac{n L}{L+1}$. In particular, the following result has been proved in [12] (see also [11]).

Theorem 1.1 ([12, Theorem 3.2]). Let $1 \leq p<\frac{n L}{L+1}$. Then, for any $\epsilon>0$ and any piece-wise affine map $\varphi \in W^{1, p}\left(\Omega ; \mathbf{R}^{n}\right)$, there exists a solution $u \in W^{1, p}\left(\Omega ; \mathbf{R}^{n}\right)$ of (1.1) satisfying $\|u-\varphi\|_{L^{p}(\Omega)}<\epsilon$.

Here, and throughout the present paper, a map $\varphi: \Omega \rightarrow \mathbf{R}^{n}$ is said to be piecewise affine if there exists a countable family of disjoint open sets $\Omega_{j} \subset \Omega$ such that

$$
\left.\varphi\right|_{\Omega_{j}}=\xi_{j} x+b_{j}, \quad\left|\Omega \backslash \bigcup_{j=1}^{\infty} \Omega_{j}\right|=0 .
$$

This theorem asserts that the boundary map $\varphi$ of very weakly quasiregular maps can be any affine map $\varphi=\xi x+b$. However, this cannot be the case if we study the usual quasiregular maps in $W^{1, n}\left(\Omega ; \mathbf{R}^{n}\right)$.

We have the following

Theorem 1.2. Given any affine map $\xi x+b$, the Dirichlet problem

$$
\left\{\begin{array}{l}
|D u(x)|^{n}=L \operatorname{det} D u(x), \quad \text { a.e. } x \in \Omega, \\
\left.u\right|_{\partial \Omega}=\xi x+b
\end{array}\right.
$$

is solvable in $W^{1, n}\left(\Omega ; \mathbf{R}^{n}\right)$ if and only if $|\xi|^{n} \leq L \operatorname{det} \xi$.

The necessary part of this result follows easily from integrating the equation and using the boundary condition and property of determinants; see also [11, Theorem 1.1] for a stronger result in $W^{1, p}\left(\Omega ; \mathbf{R}^{n}\right)$ with $p \geq n-\epsilon$.

The sufficient part of the theorem will be a special case of the main result of our present paper. (See Theorem 1.4 below.)

In order to state our main theorem, we introduce some notation. Given $L \geq 1$, consider the sets

$$
\begin{aligned}
& Z=Z_{L}=\left\{\left.\xi \in \mathbb{M}^{n \times n}|| \xi\right|^{n}=L \operatorname{det} \xi\right\}, \\
& U=U_{L}=\left\{\left.\xi \in \mathbb{M}^{n \times n}|| \xi\right|^{n}<L \operatorname{det} \xi\right\}, \\
& K=K_{L}=\left\{\left.\xi \in \mathbb{M}^{n \times n}|| \xi\right|^{n} \leq L \operatorname{det} \xi\right\},
\end{aligned}
$$

where $\mathbb{M}^{n \times n}$ denotes the space of $n \times n$ matrices with operator norm

$$
|\xi|=\max \left\{|\xi h|\left|h \in \mathbf{R}^{n},\right| h \mid=1\right\} .
$$

When $L=1$, it is easily seen that $U_{1}=\emptyset$ and $K_{1}=Z_{1}$ coincide with the set of conformal matrices, that is,

$$
K_{1}=Z_{1}=\{\lambda R \mid \lambda \geq 0, R \in S O(n)\} .
$$

In the following, we shall always assume $L>1$, thus $U=U_{L} \neq \emptyset$. 
Definition 1.1. Let $\psi \in W^{1, p}\left(\Omega ; \mathbf{R}^{n}\right)$. Define $A_{\psi}^{1, p}(\Omega ; U)$ to be the closure in the $L^{p}$-norm of the set of piece-wise affine maps $u \in \psi+W_{0}^{1, p}\left(\Omega ; \mathbf{R}^{n}\right)$ that satisfy $D u(x) \in U$ for almost every $x \in \Omega$.

Remarks. 1) Since we use the $L^{p}$-closure, unlike the space used in [4, our set $A_{\psi}^{1, p}(\Omega ; U)$ is only a closed subspace of $L^{p}\left(\Omega ; \mathbf{R}^{n}\right)$ and it may be empty, for example, if $p \geq n$ and $\psi=\xi x$ with $\xi \notin K$.

2) Clearly, we have $\psi \in A_{\psi}^{1, p}(\Omega ; U) \neq \emptyset$ if $\psi$ is itself a piece-wise affine map in $W^{1, p}\left(\Omega ; \mathbf{R}^{n}\right)$ satisfying $D \psi(x) \in U$ for almost every $x \in \Omega$.

3) We shall prove later that, for $p \geq n$, the set $A_{\psi}^{1, p}(\Omega ; U)$ is actually a subset of $\psi+W_{0}^{1, n}\left(\Omega ; \mathbf{R}^{n}\right)$ with bounded $W^{1, n}$-norm. (See Proposition 2.3 and estimate (2.9) in $\S 2$. )

The main result of this paper is the following

Theorem 1.3. Let $L>1$ and $\psi \in W^{1, n}\left(\Omega ; \mathbf{R}^{n}\right)$. Assume $A_{\psi}^{1, n}(\Omega ; U) \neq \emptyset$. Then, for any $\epsilon>0$ and any $\varphi \in A_{\psi}^{1, n}(\Omega ; U)$, there exists a map $u \in A_{\psi}^{1, n}(\Omega ; U)$ satisfying

$$
\|u-\varphi\|_{L^{n}(\Omega)}<\epsilon, \quad|D u(x)|^{n}=L \operatorname{det} D u(x) \quad \text { a.e. } x \in \Omega .
$$

Our next result, which follows readily from Theorem 1.3 generalizes the sufficient part of Theorem 1.2 (compare also with Theorem 1.1).

Theorem 1.4. For any $\epsilon>0$ and any piece-wise affine map $\varphi \in W^{1, n}\left(\Omega ; \mathbf{R}^{n}\right)$ satisfying $|D \varphi(x)|^{n} \leq L \operatorname{det} D \varphi(x)$ a.e. in $\Omega$, there exists a solution $u \in W^{1, n}\left(\Omega ; \mathbf{R}^{n}\right)$ of (1.1) satisfying $\|u-\varphi\|_{L^{n}(\Omega)}<\epsilon$.

In Yan 12, the proof of Theorem 1.1] has relied on an important technique developed in Yan [10, 11] using the idea of convex integration motivated by the work of Müller \& Šverák [7] (see also Müller \& Sychev [8]).

In this paper, we shall exploit the idea of Baire's category method as explored in the papers of Dacorogna \& Marcellini 3, 4 to prove the main result Theorem 1.3 .

However, since the sets $K=K_{L}$ and $Z=Z_{L}$ are unbounded, none of the results in papers [3, 4, 7, 8] mentioned above will work directly for our theorems and, as we shall see later, constructions leading to the Baire's category method in our proof are quite different from those given in [3, 4].

The proof we present here is elementary and does not require any notion of polyconvexity, quasiconvexity or rank-one convexity in the vectorial calculus of variations, as required in [3, 4]. However, to avoid using quasiconvexity and the related powerful lower semicontinuity theorems as given in Acerbi \& Fusco [1], in some part of the proof, we will need to rely on some higher regularity result for quasiregular mappings [5] and weak convergence result for the determinant [2, 9].

Finally, to motivate how the Baire's category method comes into play in solving our problem, let us define the solution set in Theorem 1.3 to be the set

$$
S_{\psi}^{1, n}(\Omega ; Z)=\left\{u \in A_{\psi}^{1, n}(\Omega ; U) \mid D u(x) \in Z \text { a.e. } x \in \Omega\right\} .
$$

Then Theorem 1.3 is equivalent to the following

Theorem 1.5. $S_{\psi}^{1, n}(\Omega ; Z)$ is dense in $A_{\psi}^{1, n}(\Omega ; U)$ in the $L^{n}$-metric.

We set up the problem in the frame-work of Baire's category method in \$2 A critical requirement in using the Baire's category theorem, that is, the density of certain open sets, is fulfilled in $\$ 3$ Finally, our main results are proved in the last section, \$4. 


\section{The Setting of BAiRe's CATEgory Method}

We shall use the following version of Baire's category theorem, the proof of which, given below, is elementary and included here for the convenience of the reader.

Theorem 2.1. Let $(X, \rho)$ be a nonempty complete metric space. Suppose $\left\{V_{k}\right\}$ is a family of open and dense sets in $(X, \rho)$. Then the intersection set $S=\bigcap_{k=1}^{\infty} V_{k}$ is also dense in $(X, \rho)$ and thus is nonempty.

Proof. Suppose not. Then the set $G=X \backslash \bar{S} \neq \emptyset$ is an open subset of $(X, \rho)$. We use the notation:

$$
\begin{aligned}
B(a, r) & =\{x \in X \mid \rho(x, a)<r\}, \\
B[a, r] & =\{x \in X \mid \rho(x, a) \leq r\} .
\end{aligned}
$$

Then $B(a, r)$ is an open set in $(X, \rho)$ and its closure $\overline{B(a, r)}$ is contained in the closed ball $B[a, r]$.

Since $G \neq \emptyset$ is open, we assume $B\left(a_{0}, r_{0}\right) \subset G$ for some $a_{0}$ and $r_{0}$. Since $V_{1}$ is dense and open, it follows that the open set $V_{1} \cap B\left(a_{0}, r_{0}\right)$ is nonempty; therefore, there exist $a_{1} \in V_{1} \cap B\left(a_{0}, r_{0}\right)$ and $r_{1} \in\left(0, r_{0} / 2\right)$ such that $B\left[a_{1}, r_{1}\right] \subset V_{1} \cap B\left(a_{0}, r_{0}\right)$. Inductively, we have $a_{k+1} \in V_{k+1} \cap B\left(a_{k}, r_{k}\right)$ and $r_{k+1} \in\left(0, r_{k} / 2\right)$ such that

$$
B\left[a_{k+1}, r_{k+1}\right] \subset V_{k+1} \cap B\left(a_{k}, r_{k}\right), \quad k=0,1,2, \ldots .
$$

This implies the sequence $\left\{a_{k}\right\}$ is a Cauchy sequence in $(X, \rho)$ as one has, by (2.3),

$$
\rho\left(a_{k+1}, a_{k}\right)<r_{k}<\frac{r_{0}}{2^{k}}, \quad \forall k=0,1,2, \ldots
$$

Since $(X, \rho)$ is a complete metric space, we have $a_{k} \rightarrow \bar{a}$ as $k \rightarrow \infty$ for some $\bar{a} \in X$. But, since $a_{k} \in B\left(a_{j}, r_{j}\right)$ for all $1 \leq j \leq k$, one has $\bar{a} \in B\left[a_{j}, r_{j}\right]$ for all $j=1,2, \ldots$; therefore, by (2.3) again, $\bar{a} \in \bigcap_{k=1}^{\infty} V_{k}=S$. However, since $\bar{a} \in B\left[a_{1}, r_{1}\right] \subset V_{1} \cap B\left(a_{0}, r_{0}\right)$, one has $\bar{a} \in G$, which is a contradiction with $\bar{a} \in S \subset X \backslash G$. The proof is finished.

We now set up the frame-work of using this Baire's category theorem to prove our main result as formulated in the form of Theorem 1.5.

Let $\psi \in W^{1, n}\left(\Omega ; \mathbf{R}^{n}\right)$ be a given map such that

$$
X=A_{\psi}^{1, n}(\Omega ; U) \neq \emptyset .
$$

Since $X$ is an $L^{n}$-closure, we easily have the following

Lemma 2.2. $\left(X, L^{n}\right)$ is a complete metric space.

It appears that $X$ is only a closed subspace of $L^{n}\left(\Omega ; \mathbf{R}^{n}\right)$. However, the following result asserts that $X$ is actually more "regular" than it appears.

Proposition 2.3. $X \subset\left\{\varphi \in \psi+W_{0}^{1, n}\left(\Omega ; \mathbf{R}^{n}\right) \mid D \varphi(x) \in K\right\}$.

Proof. Let $\varphi \in A_{\psi}^{1, n}(\Omega ; U)$. Then, by definition, there exists a sequence $\left\{u_{j}\right\}$ of piece-wise affine maps in $\psi+W_{0}^{1, n}\left(\Omega ; \mathbf{R}^{n}\right)$ satisfying

$$
\begin{gathered}
\left|D u_{j}(x)\right|^{n}<L \operatorname{det} D u_{j}(x), \\
\lim _{j \rightarrow \infty}\left\|u_{j}-\varphi\right\|_{L^{n}(\Omega)}=0 .
\end{gathered}
$$


Since $\left.u_{j}\right|_{\partial \Omega}=\psi$, integration of (2.5) over $\Omega$ implies

$$
\int_{\Omega}\left|D u_{j}(x)\right|^{n} d x \leq L \int_{\Omega} \operatorname{det} D \psi(x) d x \equiv M_{\psi}<\infty .
$$

Therefore, $\left\{u_{j}\right\}$ has a subsequence (labelled the same) that converges weakly to some $\bar{\varphi}$ in $\psi+W_{0}^{1, n}\left(\Omega ; \mathbf{R}^{n}\right)$ and hence converges to $\bar{\varphi}$ in the $L^{n}$-norm. On the other hand, $u_{j} \rightarrow \varphi$ in the $L^{n}$-norm, thus $\varphi=\bar{\varphi}$. This shows $\varphi \in \psi+W_{0}^{1, n}\left(\Omega ; \mathbf{R}^{n}\right)$ and hence $A_{\psi}^{1, n}(\Omega ; U) \subset \psi+W_{0}^{1, n}\left(\Omega ; \mathbf{R}^{n}\right)$.

Finally, we prove $D \varphi(x) \in K$ a.e. in $\Omega$ by using some regularity result for quasiregular mappings and weak convergence of the determinant; we could also prove this using a lower semicontinuity theorem in Acerbi \& Fusco [1, but we choose not to use that theorem here as we try to avoid the quasiconvexity condition required in the paper [1].

To prove $D \varphi(x) \in K$ a.e. in $\Omega$, we note that, by the well-known theorem of Gehring [5], maps satisfying (2.5) belong to $W_{\text {loc }}^{1, n+\epsilon}\left(\Omega ; \mathbf{R}^{n}\right)$ for some $\epsilon>0$; in fact, one has

$$
\sup _{j=1,2, \ldots} \int_{\Omega^{\prime}}\left|D u_{j}(x)\right|^{n+\epsilon} d x \leq C\left(\Omega^{\prime}\right)<\infty, \quad \forall \Omega^{\prime} \subset \subset O m .
$$

Since the determinant is weakly continuous in space $W^{1, n+\epsilon}\left(\Omega^{\prime} ; \mathbf{R}^{n}\right)$ (see [2, 9] $)$, it therefore follows that

$$
\begin{aligned}
\int_{\Omega^{\prime}}|D \varphi|^{n} d x & \leq \liminf _{j \rightarrow \infty} \int_{\Omega^{\prime}}\left|D u_{j}\right|^{n} d x \\
& \leq L \liminf _{j \rightarrow \infty} \int_{\Omega^{\prime}} \operatorname{det} D u_{j} d x=L \int_{\Omega^{\prime}} \operatorname{det} D \varphi d x
\end{aligned}
$$

for all $\Omega^{\prime} \subset \subset \Omega$. Hence, $|D \varphi(x)|^{n} \leq L \operatorname{det} D \varphi(x)$, that is, $D \varphi(x) \in K$, for almost every $x \in \Omega$.

The proof is now completed.

From the proof above, we have also proved that

$$
\int_{\Omega}|D \varphi(x)|^{n} d x \leq M_{\psi}<\infty, \quad \forall \varphi \in A_{\psi}^{1, n}(\Omega ; U) .
$$

Let

$$
F(\xi)=|\xi|^{n}-L \operatorname{det} \xi
$$

Then the sets defined by (1.3)-(1.5) are given by

$$
\begin{aligned}
& Z=\left\{\xi \in \mathbb{M}^{n \times n} \mid F(\xi)=0\right\}, \\
& U=\left\{\xi \in \mathbb{M}^{n \times n} \mid F(\xi)<0\right\}, \\
& K=\left\{\xi \in \mathbb{M}^{n \times n} \mid F(\xi) \leq 0\right\} .
\end{aligned}
$$

Since $X \subset \psi+W_{0}^{1, n}\left(\Omega ; \mathbf{R}^{n}\right)$, we can thus define the following sets, for $k=$ $1,2, \ldots$ :

$$
V_{k}=\left\{v \in X \mid \int_{\Omega} F(D v(x)) d x>-1 / k\right\} .
$$

Lemma 2.4. $V_{k}$ is open in $\left(X, L^{n}\right)$. 
Proof. It suffices to prove the complement set $C=X \backslash V_{k}$ is closed. Therefore, suppose $v_{j} \in C$ and $v_{j} \rightarrow v$ in $L^{n}\left(\Omega ; \mathbf{R}^{n}\right)$. We need to show $v \in C$. By definition, $v \in X$. Since $v_{j} \in X \backslash V_{k}$, it follows that

$$
\int_{\Omega} F\left(D v_{j}(x)\right) d x \leq-1 / k, \quad \forall j=1,2, \ldots
$$

Also, by (2.9) , there exists a subsequence of $\left\{v_{j}\right\}$ (also labelled the same) weakly convergent to $\bar{v}$ in $W^{1, n}\left(\Omega ; \mathbf{R}^{n}\right)$; this implies $v=\bar{v} \in \psi+W_{0}^{1, n}\left(\Omega ; \mathbf{R}^{n}\right)$. Therefore, by the lower semicontinuity of the $L^{n}$-norm,

$$
\begin{aligned}
\int_{\Omega} F(D v(x)) d x & =\int_{\Omega}|D v(x)|^{n} d x-L \int_{\Omega} \operatorname{det} D v(x) d x \\
& \leq \liminf _{j \rightarrow \infty} \int_{\Omega}\left|D v_{j}\right|^{n} d x-L \int_{\Omega} \operatorname{det} D \psi d x \\
& =\liminf _{j \rightarrow \infty} \int_{\Omega}\left[F\left(D v_{j}\right)+L \operatorname{det} D v_{j}\right] d x-L \int_{\Omega} \operatorname{det} D \psi d x \\
& \leq \liminf _{j \rightarrow \infty} \int_{\Omega} F\left(D v_{j}\right) d x+L \limsup _{j \rightarrow \infty} \int_{\Omega} \operatorname{det} D v_{j} d x-L \int_{\Omega} \operatorname{det} D \psi d x \\
& =\liminf _{j \rightarrow \infty} \int_{\Omega} F\left(D v_{j}(x)\right) d x \leq-1 / k \text { by (2.15). }
\end{aligned}
$$

This shows $v \notin V_{k}$, thus $v \in C$, proving $C=X \backslash V_{k}$ is closed, and hence $V_{k}$ is open in $\left(X, L^{n}\right)$.

The heart of the matter of using the Baire's category method is to establish the density of the sets $V_{k}$, which we shall do in the next section.

\section{The Density of $V_{k}$ IN $\left(X, L^{n}\right)$}

Let $X$ and $V_{k}$ be defined as above. This section is devoted to the proof of the following crucial result for the Baire's category method.

Theorem 3.1. $V_{k}$ is dense in $\left(X, L^{n}\right)$.

Proof. Assume $k \in \mathbb{N}$. Let $v \in X$ and $\epsilon>0$ be given. We need to show there exists $u=u^{\epsilon} \in V_{k}$ such that $\|u-v\|_{L^{n}(\Omega)}<\epsilon$. This amounts to finding $u^{\epsilon} \in X$ satisfying

$$
\begin{gathered}
\int_{\Omega} F\left(D u^{\epsilon}(x)\right) d x>-1 / k, \\
\left\|u^{\epsilon}-v\right\|_{L^{n}(\Omega)}<\epsilon .
\end{gathered}
$$

Since $v \in X$, we can select a piece-wise affine map $v_{1} \in \psi+W_{0}^{1, n}\left(\Omega ; \mathbf{R}^{n}\right)$ satisfying

$$
\left\|v-v_{1}\right\|_{L^{n}(\Omega)}<\epsilon / 2, \quad v_{1}=\sum_{j=1}^{\infty}\left(\xi_{j} x+b_{j}\right) \chi_{\Omega_{j}},
$$

with $\chi_{S}$ denoting the characteristic function of set $S$, where $\Omega_{j}$ are disjoint open sets in $\Omega$ and $\xi_{j} \in U$ such that

$$
\left|\Omega \backslash \bigcup_{j=1}^{\infty} \Omega_{j}\right|=0, \quad \sum_{j=1}^{\infty}\left|\xi_{j}\right|^{n}\left|\Omega_{j}\right|=\left\|D v_{1}\right\|_{L^{n}(\Omega)}^{n}<\infty .
$$


Before continuing the proof, we prove the following useful result.

Proposition 3.2. Let $\Sigma \subset \mathbf{R}^{n}$ be a bounded open set. Assume

$$
\left|\xi_{0}\right|^{n}<L \operatorname{det} \xi_{0}<\lambda \text {. }
$$

Then, for any $b_{0} \in \mathbf{R}^{n}$ and any $\delta>0$, there exists a piece-wise affine map $w=$ $w^{\delta} \in\left(\xi_{0} x+b_{0}\right)+W_{0}^{1, \infty}\left(\Sigma ; \mathbf{R}^{n}\right)$ such that

$$
\left\{\begin{array}{l}
|D w(x)|^{n}<L \operatorname{det} D w(x)<\lambda \quad \text { a.e. } x \in \Sigma \\
\left\|w-\left(\xi_{0} x+b_{0}\right)\right\|_{L^{\infty}(\Sigma)}<\delta \\
\int_{\Sigma} \operatorname{dist}^{n}(D w(x) ; Z) d x<\delta .
\end{array}\right.
$$

Proof. Step 1. We find $R, Q \in S O(n)$ such that

$$
\xi_{0}=R\left(\begin{array}{cccc}
\epsilon_{n} & & & 0 \\
& \epsilon_{n-1} & & \\
& & \ddots & \\
0 & & & \epsilon_{1}
\end{array}\right) Q \equiv R \tilde{\xi}_{0} Q,
$$

where $0<\epsilon_{1} \leq \epsilon_{2} \leq \cdots \leq \epsilon_{n-1} \leq \epsilon_{n}$ satisfy

$$
\epsilon_{n}^{n}<L \epsilon_{1} \epsilon_{2} \cdots \epsilon_{n}<\lambda
$$

Let $\widetilde{\Sigma}=Q \Sigma$ and $\tilde{b}_{0}=R^{T} b_{0}$.

Step 2. We claim there exists a piece-wise affine map $\tilde{w}(y) \in\left(\tilde{\xi}_{0} y+\tilde{b}_{0}\right)+$ $W_{0}^{1, \infty}\left(\widetilde{\Sigma} ; \mathbf{R}^{n}\right)$ such that

$$
\left\{\begin{array}{l}
|D \tilde{w}(y)|^{n}<L \operatorname{det} D \tilde{w}(y)<\lambda \quad \text { a.e. } y \in \widetilde{\Sigma} \\
\left\|\tilde{w}-\left(\tilde{\xi}_{0} y+\tilde{b}_{0}\right)\right\|_{L^{\infty}(\widetilde{\Sigma})}<\delta, \\
\int_{\widetilde{\Sigma}} \operatorname{dist}^{n}(D \tilde{w}(y) ; Z) d y<\delta .
\end{array}\right.
$$

Assuming this claim, we define $w(x)=R \tilde{w}(Q x)$ for $x \in \Sigma$. It can be easily shown that the map $w=w(x)$ satisfies all requirements of the proposition and the proof is completed.

Step 3. We now prove the claim of Step 2. We start with the matrix

$$
\eta=\eta(t)=\left(\begin{array}{cccc}
\epsilon_{n} & t & & 0 \\
0 & \epsilon_{n-1} & & 0 \\
& & \ddots & \\
0 & 0 & & \epsilon_{1}
\end{array}\right), \quad t \in \mathbf{R} .
$$

It can be easily seen that $\operatorname{det} \eta(t)=\epsilon_{1} \epsilon_{2} \cdots \epsilon_{n}$ for all $t \in \mathbf{R}$ and

$$
f(t)=|\eta(t)|^{n}=\left(\frac{\epsilon_{n}^{2}+\epsilon_{n-1}^{2}+t^{2}+\sqrt{\left(\epsilon_{n}^{2}+\epsilon_{n-1}^{2}+t^{2}\right)^{2}-4 \epsilon_{n}^{2} \epsilon_{n-1}^{2}}}{2}\right)^{\frac{n}{2}} .
$$

Note that $f(0)=\epsilon_{n}^{n}<L \epsilon_{1} \cdots \epsilon_{n}<\lambda$ and $f(t) \rightarrow \infty$ as $|t| \rightarrow \infty$. Therefore, there exists a (unique) $t_{0}>0$ such that

$$
f(t)<f\left( \pm t_{0}\right)=L \epsilon_{1} \cdots \epsilon_{n}, \quad \forall t \in\left(-t_{0}, t_{0}\right) .
$$

This implies

$$
\eta^{ \pm}=\eta\left( \pm t_{0}\right) \in Z, \quad \eta(t) \in U^{\lambda}, \quad \forall t \in\left(-t_{0}, t_{0}\right)
$$


where

$$
U^{\lambda}=\left\{\left.\xi \in \mathbb{M}^{n \times n}|| \xi\right|^{n}<L \operatorname{det} \xi<\lambda\right\} \subset U
$$

is a bounded open set in $\mathbb{M}^{n \times n}$.

Let $0<\gamma<\min \left\{\delta, t_{0}\right\}$ be any given number. Let $\eta_{1}=\eta\left(-t_{0}+\gamma\right)$ and $\eta_{2}=$ $\eta\left(t_{0}-\gamma\right)$. Then, since $\eta^{ \pm} \in Z$, it follows that

$$
\eta_{k} \in U^{\lambda}, \quad \operatorname{dist}\left(\eta_{k} ; Z\right) \leq\left|\eta_{1}-\eta^{-}\right|=\left|\eta_{2}-\eta^{+}\right|=\gamma \quad(k=1,2)
$$

and

$$
\tilde{\xi}_{0}=\frac{1}{2}\left(\eta_{1}+\eta_{2}\right) \in U^{\lambda} .
$$

We are now in a position to use the following result.

Lemma 3.3. Let $G$ be an open set in $\mathbb{M}^{m \times n}$ and let $\eta \in G$ and $\eta=t \eta_{1}+(1-t) \eta_{2}$ with $t \in(0,1)$ and $\operatorname{rank}\left(\eta_{1}-\eta_{2}\right)=1$. Then, for any $b \in \mathbf{R}^{m}$ and any $\gamma>0$, there exists a piece-wise affine map $u \in(\eta x+b)+W_{0}^{1, \infty}\left(\Omega ; \mathbf{R}^{m}\right)$ such that

$$
\left\{\begin{array}{l}
D u(x) \in\left\{\eta_{1}, \eta_{2}, \eta_{3}, \ldots, \eta_{s}\right\} \quad \text { a.e. } x \in \Omega, \\
\left\{\eta_{3}, \ldots, \eta_{s}\right\} \subset G, \\
\|u-(\eta x+b)\|_{L^{\infty}(\Omega)}<\gamma \\
\left|\left\{x \in \Omega \mid D u(x) \notin\left\{\eta_{1}, \eta_{2}\right\}\right\}\right|<\gamma .
\end{array}\right.
$$

Proof. Except for the requirement $\|u-(\eta x+b)\|_{L^{\infty}(\Omega)}<\gamma$, the result is exactly the same as [11, Lemma 3.4]; however, the requirement $\|u-(\eta x+b)\|_{L^{\infty}(\Omega)}<\gamma$ follows from [11, Lemma 3.1] of that paper.

Using this lemma, with $\eta=\tilde{\xi}_{0}, G=U^{\lambda}$ and $\Omega=\widetilde{\Sigma}$, we find a piece-wise affine $\operatorname{map} \tilde{w}=\tilde{w}_{\gamma}(y) \in\left(\tilde{\xi}_{0} y+\tilde{b}_{0}\right)+W_{0}^{1, \infty}\left(\widetilde{\Sigma} ; \mathbf{R}^{n}\right)$ satisfying

$$
\left\{\begin{array}{l}
D \tilde{w}(y) \in U^{\lambda} \quad \text { a.e. } y \in \widetilde{\Sigma}, \\
\left\|\tilde{w}-\left(\tilde{\xi}_{0} y+\tilde{b}_{0}\right)\right\|_{L^{\infty}(\widetilde{\Sigma})}<\gamma, \\
\left|\left\{y \in \widetilde{\Sigma} \mid D \tilde{w}(y) \notin\left\{\eta_{1}, \eta_{2}\right\}\right\}\right|<\gamma .
\end{array}\right.
$$

Hence, we have the estimate

$$
\begin{aligned}
& \int_{\tilde{\Sigma}} \operatorname{dist}^{n}(D \tilde{w}(y) ; Z) d y \\
& \quad=\int_{D \tilde{w}(y) \in\left\{\eta_{1}, \eta_{2}\right\}} \operatorname{dist}^{n}(D \tilde{w} ; Z) d y+\int_{D \tilde{w}(y) \notin\left\{\eta_{1}, \eta_{2}\right\}} \operatorname{dist}^{n}(D \tilde{w} ; Z) d y \\
& \quad \leq \gamma^{n}|\widetilde{\Sigma}|+\gamma \lambda<\delta
\end{aligned}
$$

if $\gamma$ is sufficiently small, where we have used (3.9) and the fact that $\operatorname{dist}^{n}(\eta ; Z) \leq$ $|\eta|^{n}<\lambda$ for all $\eta \in U^{\lambda}$.

Consequently, this $\tilde{w}(y)$ satisfies the claim in Step 2. This completes Step 3 and the proof of Proposition 3.2

We now continue the proof of Theorem [3.1] For each given $\xi_{j}, \Omega_{j}$ as given in (3.3) - 3.4), we apply Proposition 3.2 with $\xi_{0}=\xi_{j}, b_{0}=b_{j}, \lambda=2 L\left|\xi_{j}\right|^{n}, \Sigma=\Omega_{j}$ and $\delta=\rho / 2^{j}, 0<\rho<\epsilon$ being a number to be selected later, to obtain a piece-wise 
affine map $u_{j}^{\rho} \in\left(\xi_{j} x+b_{j}\right)+W_{0}^{1, \infty}\left(\Omega_{j} ; \mathbf{R}^{n}\right)$ satisfying

$$
\left\{\begin{array}{l}
\left|D u_{j}^{\rho}(x)\right|^{n}<L \operatorname{det} D u_{j}^{\rho}(x)<2 L\left|\xi_{j}\right|^{n} \quad \text { a.e. } x \in \Sigma \\
\left\|u_{j}^{\rho}-\left(\xi_{j} x+b_{j}\right)\right\|_{L^{\infty}\left(\Omega_{j}\right)}<\rho / 2^{j}<\rho, \\
\int_{\Omega_{j}} \operatorname{dist}^{n}\left(D u_{j}^{\rho}(x) ; Z\right) d x<\rho / 2^{j}
\end{array}\right.
$$

Let

$$
v^{\rho}=\sum_{j=1}^{\infty} u_{j}^{\rho} \chi_{\Omega_{j}} .
$$

Then $v^{\rho}$ is a piece-wise affine map in $\psi+W_{0}^{1, n}\left(\Omega ; \mathbf{R}^{n}\right)$ (see, e.g., [11, Lemma 1.6]) and satisfies $D v^{\rho}(x) \in U$ for almost every $x \in \Omega$. Therefore, $v^{\rho} \in X$. We also have

$$
\begin{aligned}
\left\|v^{\rho}-v_{1}\right\|_{L^{n}(\Omega)} & \leq \rho|\Omega|^{1 / n}, \\
\int_{\Omega} \operatorname{dist}^{n}\left(D v^{\rho} ; Z\right) d x & \leq \sum_{j=1}^{\infty} \frac{\rho}{2^{j}}=\rho, \\
\int_{\Omega}\left|D v^{\rho}(x)\right|^{n} d x & =\sum_{j=1}^{\infty} \int_{\Omega_{j}}\left|D u_{j}^{\rho}(x)\right|^{n} d x \\
& \leq \sum_{j=1}^{\infty} 2 L\left|\xi_{j}\right|^{n}\left|\Omega_{j}\right|=2 L\left\|D v_{1}\right\|_{L^{n}(\Omega)}^{n} .
\end{aligned}
$$

The following useful result is elementary.

Lemma 3.4. For each $\tau>0$ there exists a constant $C_{\tau}>0$ such that

$$
F(\xi)>-\tau|\xi|^{n}-C_{\tau} \operatorname{dist}^{n}(\xi ; Z), \quad \forall \xi \in \mathbb{M}^{n \times n} .
$$

Proof. Since both $F(\xi)$ and $\operatorname{dist}^{n}(\xi ; Z)$ are homogeneous of degree $n$, one has only to prove the stated inequality for all $\xi$ with $|\xi|=1$. We use the contradiction method. Suppose, for the contrary, the inequality does not hold for $|\xi|=1$. Then, for some $\tau_{0}>0$ and all integers $j=1,2, \ldots$, there exists $\xi_{j} \in \mathbb{M}^{n \times n}$ with $\left|\xi_{j}\right|=1$ such that

$$
F\left(\xi_{j}\right) \leq-\tau_{0}-j \operatorname{dist}^{n}\left(\xi_{j} ; Z\right), \quad \forall j=1,2, \ldots .
$$

Without loss of generality, assume $\xi_{j} \rightarrow \bar{\xi}$ for some $\bar{\xi}$ with $|\bar{\xi}|=1$ as $j \rightarrow \infty$. Since $F\left(\xi_{j}\right) \rightarrow F(\bar{\xi})$ and $\operatorname{dist}^{n}\left(\xi_{j} ; Z\right) \rightarrow \operatorname{dist}^{n}(\bar{\xi} ; Z)$ as $j \rightarrow \infty$, it follows from (3.17) that $\operatorname{dist}^{n}(\bar{\xi} ; Z) \leq 0$, thus, $\bar{\xi} \in Z$ and $F(\bar{\xi})=0$. However, (3.17) also implies $F\left(\xi_{j}\right) \leq-\tau_{0}$ for all $j$ so that $F(\bar{\xi}) \leq-\tau_{0}$, which contradicts $F(\bar{\xi})=0$. The lemma is proved.

Using this lemma, we have by (3.14), (3.15), 3.16) that

$$
\begin{aligned}
\int_{\Omega} F\left(D v^{\rho}(x)\right) d x \geq & -\tau \int_{\Omega}\left|D v^{\rho}(x)\right|^{n} d x \\
& -C_{\tau} \int_{\Omega} \operatorname{dist}^{n}\left(D v^{\rho}(x) ; Z\right) d x \\
\geq & -2 L \tau\left\|D v_{1}\right\|_{L^{n}(\Omega)}^{n}-C_{\tau} \rho .
\end{aligned}
$$

To complete the proof, we select $\tau>0$ such that

$$
2 L \tau\left\|D v_{1}\right\|_{L^{n}(\Omega)}^{n}<\frac{1}{2 k}
$$


and, for this $\tau>0$, select $\rho>0$ such that

$$
C_{\tau} \rho<\frac{1}{2 k}, \quad \rho|\Omega|^{1 / n}<\epsilon / 2 .
$$

Let $u^{\epsilon}=v^{\rho}$. Then, from (3.20), we easily see

$$
\int_{\Omega} F\left(D u^{\epsilon}(x)\right) d x>-1 / k
$$

(hence $u^{\epsilon} \in V_{k}$ ) and, by (3.3), (3.13),

$$
\begin{aligned}
\left\|u^{\epsilon}-v\right\|_{L^{n}(\Omega)} & \leq\left\|v^{\rho}-v_{1}\right\|_{L^{n}(\Omega)}+\left\|v_{1}-v\right\|_{L^{n}(\Omega)} \\
& <\epsilon / 2+\epsilon / 2=\epsilon .
\end{aligned}
$$

Theorem 3.1 is finally proved.

\section{Proof of the main theorems}

In this final section, we present the proof of our main theorems. We use the same notation as above.

Proposition 4.1. Let $S_{\psi}^{1, n}(\Omega ; Z)$ be defined by (1.7). Then

$$
S_{\psi}^{1, n}(\Omega ; Z)=\bigcap_{k=1}^{\infty} V_{k} .
$$

Proof. From definition (1.7), $S_{\psi}^{1, n}(\Omega ; Z)=\{v \in X \mid F(D v(x))=0\}$. Therefore,

$$
S_{\psi}^{1, n}(\Omega ; Z) \subset V_{k}
$$

for all $k=1,2, \ldots$, and hence $S_{\psi}^{1, n}(\Omega ; Z) \subset \bigcap_{k=1}^{\infty} V_{k}$.

On the other hand, suppose $v \in \bigcap_{k=1}^{\infty} V_{k}$. Then

$$
\int_{\Omega} F(D v(x)) d x>-1 / k, \quad \forall k=1,2, \ldots,
$$

and hence $\int_{\Omega} F(D v(x)) d x \geq 0$. By Proposition 2.3 one also has $D v(x) \in K$, i.e., $F(D v(x)) \leq 0$ a.e. in $\Omega$. Therefore, $F(D v(x))=0$ and $D v(x) \in Z$ for a.e. $x \in \Omega$, and hence $v \in S_{\psi}^{1, n}(\Omega ; Z)$. This proves $\bigcap_{k=1}^{\infty} V_{k} \subset S_{\psi}^{1, n}(\Omega ; Z)$. The result is proved.

Proof of Theorems 1.3 and 1.5. Theorem 1.5 and hence Theorem 1.3 follow from the Baire's category theorem (Theorem 2.1), using Lemmas 2.2 2.4 Theorem 3.1 and Proposition 4.1.

Proof of Theorem 1.4. There are two disjoint open subsets $\Omega_{0}$ and $\Omega_{1}$ of $\Omega$ such that $\left|\Omega \backslash\left(\Omega_{0} \cup \Omega_{1}\right)\right|=0$ satisfying

$$
D \varphi(x) \in Z, \quad \text { a.e. } x \in \Omega_{0} ; \quad D \varphi(x) \in U, \quad \text { a.e. } x \in \Omega_{1} .
$$

We use the existence result Theorem 1.3 for the boundary value $\varphi \in A_{\varphi}^{1, p}\left(\Omega_{1} ; U\right)$ on $\Omega_{1}$ to obtain a solution $u_{1} \in \varphi+W_{0}^{1, n}\left(\Omega_{1} ; \mathbf{R}^{n}\right)$ satisfying

$$
\left\|u_{1}-\varphi\right\|_{L^{n}\left(\Omega_{1}\right)}<\epsilon, \quad D u_{1}(x) \in Z \quad \text { a.e. } x \in \Omega_{1} .
$$

Then, it is easy to see that $u=u_{1} \chi_{\Omega_{1}}+\varphi \chi_{\Omega_{0}}$ is a solution required in the theorem. 


\section{REFERENCES}

[1] E. Acerbi and N. Fusco, Semicontinuity problems in the calculus of variations, Arch. Rational Mech. Anal., 86 (1984), 125-145. MR 85m:49021

[2] J. M. Ball, Convexity conditions and existence theorems in nonlinear elasticity, Arch. Rational Mech. Anal., 63 (1977), 337-403. MR 57:14788

[3] B. Dacorogna and P. Marcellini, General existence theorems for Hamilton-Jacobi equations in the scalar and vectorial cases, Acta Math., 178 (1) (1997), 1-37. MR 98d:35029

[4] B. Dacorogna and P. Marcellini, Cauchy-Dirichlet problem for first order nonlinear systems, J. Funct. Anal., 152 (2) (1998), 404-446. MR 99a:35025

[5] F. W. Gehring, The $L^{p}$-integrability of the partial derivatives of a quasiconformal mapping, Acta Math., 130 (1973), 265-277. MR 53:5861

[6] T. Iwaniec, p-Harmonic tensors and quasiregular mappings, Ann. Math., 136 (1992), 589624. MR 94d:30034

[7] S. Müller and V. Šverák, Attainment results for the two-well problem by convex integration, Geometric analysis and the calculus of variations, 239-251, Internat. Press; Cambridge, MA, 1996. MR 98e:49004

[8] S. Müller and M. A. Sychev, Optimal existence theorems for nonhomogeneous differential inclusions, J. Funct. Anal. 181 (2001), no. 2, 447-475. MR 2002c:35281

[9] Yu. G. Reshetnyak, "Space Mappings with Bounded Distortion," Transl. Math. Monograph, 73, Amer. Math. Soc., 1989. MR 90d:30067

[10] B. Yan, Linear boundary values of weakly quasiregular mappings, C. R. Acad. Sci. Paris Ser. I Math., 331 (2000), 379-384. MR 2001e:35037

[11] B. Yan, A linear boundary value problem for weakly quasiregular mappings in space, Cal. Var. Partial Differential Equations, 13 (2001), no. 3, 295-310. MR 2002h:30026

[12] B. Yan, Relaxation and attainment results for an integral functional with unbounded energywell, Proc. Roy. Soc. Edinburgh Sect. A 132 (2002), no. 6, 1513-1523.

Department of Mathematics, Michigan State University, East Lansing, Michigan 48824

E-mail address: yan@math.msu.edu 